\title{
The SAS grade: underused and under recognised?
}

\author{
S. Wilmott*1 and C. J. Mannion²
}

\section{Key points}

The Specialty and Associate Specialist (SAS) grade dentist plays an important role in the smooth running of hospital dental departments across the country.
The SAS charter should be implemented in hospital dental departments to support and develop SAS grade dentists.
The SAS grade is an additional career option for young dentists.

The speciality and associate specialist grade doctor is an important part of many specialist departments in both medicine and dentistry. In this article we highlight their vital (and often forgotten) contribution in providing and delivering excellent patient care within the dental specialties. We suggest that more should be done to address the needs of this important section of our workforce. Encouraging a programme of continued professional development together with a more structured path for career progression should be considered.

The importance of the role played by speciality and associate specialist (SAS) doctors throughout the NHS has been highlighted recently in BMJ publications. ${ }^{1,2}$ These articles act as a timely reminder of the vital contribution of the many dentists who also currently work as SAS grades in hospital departments throughout the UK.

SAS grade doctors comprise $20 \%$ of the medical workforce and this proportion is consistent for SAS dentists as a whole. ${ }^{3}$ However, SAS dentists in the speciality of oral surgery make up almost $45 \%$ of the workforce, ${ }^{3}$ which is one of the largest proportions in hospital medicine and dentistry combined. Although the data is limited, we do know that within oral and maxillofacial surgery (OMFS) and oral surgery there are 330 SAS dentists currently working in the UK. ${ }^{3}$ We feel that the contributions of this substantial group should be highlighted, and issues such as career progression should be addressed.

In OMFS, SAS posts are invaluable for gaining hands-on clinical experience and

${ }^{1}$ Speciality Dentist in Oral and Maxillofacial Surgery, Leeds Teaching Hospitals Trust; ${ }^{2}$ Consultant and Clinical Associate Professor in Oral and Maxillofacial Surgery, Leeds Teaching Hospitals Trust

${ }^{*}$ Correspondence to: Miss Sheryl Wilmott

Email: sheryl.wilmott@nhs.net

Refereed Paper. Accepted 14 September 2018 DOI: $10.1038 /$ sj.bdj.2018.870 improving the dentist's professional portfolio through teaching and involvement in clinical governance. Other reasons for working as an SAS grade include fewer administrative functions when compared to consultants and an improved work-life balance. ${ }^{4}$ Sometimes difficulties gaining a training post can 'push' dentists into SAS posts. ${ }^{4}$ Posts can be temporary or permanent and job titles such as staff grade, associate specialists, speciality dentists and hospital practitioners are used to identify their position and role.

Along with consultants and auxiliary staff, SAS dentists are a consistent presence in a department. They can establish continuity and stability in teams where the junior workforce frequently moves between posts. As a result, they are uniquely positioned to play an important role in teaching, management and quality improvement. Many undertake postgraduate qualifications, are widely published in peer-reviewed journals, and are members of royal colleges and specialist societies. Their level of experience varies from recent completion of dental core training up to and including senior clinicians involved in senior management and training within deaneries. In our department, we actively encourage our SAS colleagues to undertake diverse roles and professional development. These include being involved in the local negotiating committee, undertaking postgraduate research degrees in public health, and certificates and diplomas in medical education and conscious sedation. We believe that this varied range of clinical interests enriches our department and contributes to our overall philosophy of excellence in patient care.

However, the lack of a formal and defined path for career progression for SAS doctors and dentists, together with limited development opportunities can lead to this group feeling demoralised. They can have difficulties accessing educational opportunities due to their roles being classed as 'non-training' and they can feel and find that their contributions to their departments go unrecognised. ${ }^{5}$ Although permanent roles offer some stability, departmental rota changes tend to reflect service needs rather than the clinical interests and educational needs of SAS dentists. This lack of professional stimulation can lead to boredom, dissatisfaction and burnout. ${ }^{6}$ There are no UK data on job satisfaction in SAS dentists, but there is evidence that dentists working in oral surgery, a high proportion of whom are SAS grades, experience one of the highest levels of burnout in the dental specialties ${ }^{7,8}$ and this is an area where further research is indicated.

In February 2017 the British Medical Association released a document which aimed to ensure that best practice is applied to the development of SAS doctors and dentists. ${ }^{9}$ This followed on from the SAS charter, ${ }^{10}$ developed 
by the BMA in 2014 with input from the Academy of Medical Royal Colleges, Health Education England and NHS Employers. It sets out what SAS doctors can expect from their employers and what their employers can expect of them. Some of the suggestions include:

- Increased autonomy: this recognises the highly skilled SAS doctors and dentists that work in the NHS and gives them more responsibility where appropriate. This improves the engagement and motivation of staff which can only benefit the patients that they see

- Eligibility for specialist registration: SAS doctors who have not followed a UK specialist training programme can gain specialist status via a Certificate of Eligibility for Specialist Registration (CESR). This requires a verified portfolio of evidence which shows equivalent knowledge and experience to that of a doctor who has followed a specialist training programme. ${ }^{11}$ This route is not currently open to UK dentists, but in the meantime continued professional and educational development should be facilitated with a more defined ladder of career progression. This would develop a more positive view of SAS posts and encourage the individual to maintain and develop a robust body of evidence of their achievements

- Development and extended roles: educational supervisors and appraisers must be appropriately trained doctors and dentists, but there is no requirement for them to be on the specialist register. The permanence and stability of SAS dentists makes them ideal to take on these roles. Management and training roles can also be suitable for SAS dentists.

Support for our SAS colleagues to progress and succeed must become embedded within the culture of our specialist departments. Investing in the professional development of this group will promote a far more positive view of the SAS role. More importantly, feeling valued has considerable benefits for the team spirit of our departments and thus to patient care.

So, let's encourage our departments to implement the SAS charter. It will demonstrate a commitment to developing all team members and provide an environment in which our SAS colleagues can flourish.

1. Brinkley G. SAS doctors are a highly specialised and essential part of the workforce. BMJ 2018; (Suppl. BMA News): 3.
2. Nath V. SAS doctors are an underused resource in the NHS BMJ 2016; 353: DOI: 10.1136/bmi.i3363.

3. NHS Digital. NHS Workforce Statistics. 2017. Available at https://digital.nhs.uk/data-and-information/publications/ statistical/nhs-workforce-statistics/nhs-workforce-statistics-august-2017-provisional-statistics (accessed April 2018).

4. Royal College of Physicians. Speciality, associate specialist and staff grade (SAS) doctors. 2015. Available at https:// www.rcplondon.ac.uk/education-practice/advice/specialty-associate-specialist-and-staff-grade-sas-doctors (accessed April 2018).

5. Phazey G, Agius S, Hayden J. SAS doctors' perceptions of their role in the NHS. BMJ 2012; 344: DOI 10.1136/bmj. e2819.

6. Harju L K, Hakanen J J, Schaufeli W B. Can job crafting reduce job boredom and increase work engagement? A three-year cross-lagged panel study. J Vocat Behav 2016; 95-96: 11-20.

7. Larbie J, Kemp M, Whitehead P. The Mental Health and Well-being of UK Dentists: A Qualitative Study About the BDA. 2017. Available from: www.bda.org/burnout (accessed April 2018)

8. Humphris G, Lilley J, Kaney S, Broomfield D. Burnout and stress-related factors among junior staff of three dental hospital specialties. Br Dent J 1997; 183: 15-21.

9. Academy of Medical Royal Colleges. SAS doctor development - Summary of resources and further work. 2017. Available at http://www.aomrc.org.uk/ wp-content/uploads/2017/02/SAS_doctor_development_ guide_220217-1.pdf (accessed September 2018).

10. British Medical Association. A charter for staff and associate specialist and speciality doctors. 2014. Available at https://www.bma.org.uk/-/media/files/pdfs/developing\%20 your $\% 20$ career $/$ sas $\% 20$ grade $\% 20$ doctor/sas_charter_ england_dec2014.pdf (accessed September 2018).

11. Willis D. CESR: a guide to survival. BMJ 2013; 346: DOI: $10.1136 / \mathrm{bmj} .11228$.

12. BMA. Staff and associate specialists committee conference 2018. Available at https://www.bma.org.uk/events/2018/ may/staff-and-associate-specialists-committee-conference (accessed April 2018). 